\title{
Research on the construction of Maritime Intelligent Emergency Command System
}

\author{
Haoqiong Hu, Keyi Zhu and Hu Liu \\ Port and Transportation College of Zhejiang Ocean University, Zhoushan 316000, P. R. China
}

\begin{abstract}
The situation of emergency command about Maritime affairs at present was analyzed, and the maritime intelligent emergency command system was set up from four aspects -functional structure, intelligent pre-plan, system architecture, technical features, which shows the functional completeness, technical feasibility, simple operation and high integration features, and so on. It complies with the development trend of maritime emergency command information and intelligence.
\end{abstract}

Keyword-intelligent pre-plan; emergency command; system construction;maritime affair

\section{INTRODUCTION}

With the rapid development of the port economy, the regulatory situation about safety on water is becoming worse and worse, which leads to higher demand, greater responsibility and heavier stress for the search and rescue mission. It is mainly embodied as follows:

The quantity increase of in-and-out port ships will lead to the probability increase of emergency incident happened on water, especially international ships to accidents concerning foreign countries.

The large-scale trend of ship in-and-out port will lead to the high probability of emergency with high level on water.

The increase of the throughput about the dangerous goods in port will lead to an increase the probability of water pollution incidents happened in port.

Although lots of high technology is used in navigational area to ensure the safety of ship, such as VTS, AIS, VHF, CCTV, ship dynamic 2.0 management system, crew management system, ship registration system, information release system and so on. There is no system for the water emergencies yet, which has been unable to meet the requirement of emergency management of maritime search and rescue in the rapid development of social economy.

Therefore, to develop maritime intelligent emergency management system has become an important measure to solve the current problems. At the same time, in order to solve the problems of heavy tasks with a small number of maritime emergency workers and high pressure on the various system, the maritime intelligent emergency management system needs to be integrated to enhance the role that informatization construction should play in the entire maritime management business.

\section{CURRENT SituAtion OF MARITIME EMERGENCY COMMAND}

MSA (Maritime Safety Agency) is responsible for emergency operations, the main contents include: Business of daily management, such as daily work of maritime emergency management including daily duty of emergency, emergency resources maintenance, dangerous work report, general events disposal and so on.

Business of Emergency treatment and monitor, responsible for the emergency disposal of various dangerous tasks, including: report management and rapid disposal of emergency, disposal report management, judgment on danger level of emergency, emergency notification, power dispatching and onsite disposal records, prediction of the future dynamic development trend of event, emergency termination review, which carried out manually according to the standard of emergency handling business. At present, under the unified guidance of MSA, the modern informatization means such as VTS, AIS, GIS, GPS, CCTV, VHF and maritime communication have been fully applied, and the relevant information management system and standards have been gradually established. Especially the GIS system developed by MSA has become an important information platform for maritime daily dynamic supervision and command, which effectively integrate the relevant law enforcement information system, including the cruise rescue station, emergency standby point and other dynamic law enforcement resources, to provide a solid foundation for the construction of emergency management system. The management of the grid dynamic supervision model was full applied, which established dynamic enforcement mechanism of unified command and coordination with the command center as the core, optimized the dynamic law enforcement process, smoothed information flow channels and integrated maritime law enforcement resources to achieve a fine, scientific and informational maritime dynamic management, emergency response and the ability to effectively enhance the disposal. It provides a powerful system guarantee for the construction of emergency management assistance and emergency decision support system. Maritime emergency assistance command system has been developed by the Ministry of administration in 2007, but the system cannot provide timely response to emergency command in emergency situations due to its incline to the input and query of plans, reporting of accident risk information. At present, Maritime emergency disposal is relied on the operator in command center who has enough experience, which cannot avoid the untimely or additionally recording for people’s negligence. 


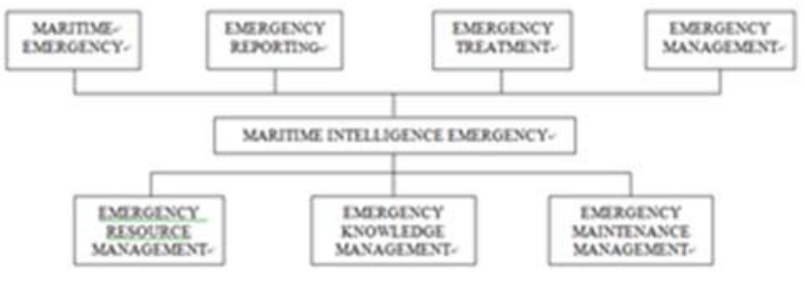

FIGURE I. THE OVERALL FUNCTIONAL STRUCTURE OF THE SYSTEM

\section{DESIGN OF INTELLIGENT EMERGENCY COMMAND SYSTEM}

According to the requirements of the National Maritime Bureau standards of emergency and emergency system construction technology, maritime intelligent emergency command system was designed by full use of existing resources, adhered to the principle of combination of peacetime and wartime, share in common, relied on the existing network system, the spatial information and service sector data, used the collaborative and sharing model, looked into all types of maritime emergency focus on emergency events, emergency command and decision analysis, in order to enhance the ability of fast reaction for emergency command, dispatch and prevention.

\section{A. Functional Structure Design}

Maritime Intelligent Emergency Command System, in order to integrate the existing emergency resources, the accident and dangerous situation of rapid and effective relief as the starting point, realized emergency command data query, search and rescue operations decision-making, search and rescue forces joint command, by means of informatization. Its decision-making is scientific and information submitted is convenient. Aimed to improve and enhance the maritime emergency organization, command, coordination, according to the design principle of intelligent plan model, combination with the work reality of maritime emergency command system and work habits of attendant in command center and VTS, the function of the system was designed in accordance with the time axis order for establishment of emergency response plan, management, model emergency report management and dispatching. The system mainly consists of seven parts: maritime emergency duty system, emergency incident receiving system, emergency disposal system, emergency plan management system, emergency resource management system, emergency knowledge management system and background maintenance management system. The overall functional structure of the system is shown in Figure 1.

\section{1) Maritime Emergency Duty System}

The maritime emergency duty system mainly realized the functions of receiving, sending, recording, tracking, reminding and querying the daily duty information between the maritime emergency command center and the emergency duty units. The system can also manage the related conferences and materials of emergency management, and analyzed the information connection and information reporting of emergencies. The main functions include: information management, duty management, address book management, conference management, material management, video duty, statistical analysis.

\section{2) Emergency Reporting System}

The emergency incident receiving system realizes the reception and reporting of emergencies. It requires information to be entered at a time to avoid repeated operation. It integrates CTI system and ship 2.0 system, integrates GIS system to achieve spatial location of emergencies location map. The professional terminology of marine emergencies is in line with the industry standards and supports the dynamic maintenance of professional terms. Reduce manual input operation and support touch screen operation. The main functions include: event information entry, communication information acquisition, ship information acquisition, location annotation, event level auxiliary research, event briefs, transfer reports, etc.

\section{3) Emergency Disposal System}

Assist emergency managers formulate solutions, effective deployment scheduling and rescue teams, emergency supplies, emergency equipment and other resources, real-time or timely development of public emergencies and emergency situations passed to the relevant personnel, to achieve coordinated command, orderly scheduling and effective supervision, improve the efficiency of emergency. The main functions include: Summarization of events, increase of danger, increase of disposal steps, automatic generation of disposal steps, adjustment of disposal steps, allocation of resources, instruction, command feedback, event secondary disaster report and disposition report generation.

\section{4) Emergency Plan Management System}

The system realized emergency response plan and the special plan preparation, maintenance, resolution, query, through the effective integration of the area plan, public emergency management organization and information resources such as water, clear water to deal with public emergencies prevention and disposal process, command, and the emergency plan expressed by picture, paper and dynamic interaction way in the system. The main functions include: plan preparation, pre -plan release, pre-plan revision, pre-plan inquiry, pre-plan decomposition, pre-plan resource management, and pre-plan Association.

\section{5) Emergency Resource Management System}

The emergency resource management system should realize dynamic management of emergency resources such as patrol boat, rescue team, reserve material, rescue equipment, communication guarantee and so on, so as to provide guarantee for emergency command and operation. It provides map annotation function for emergency resources, forms thematic map of emergency resources, realizes dynamic management of emergency resources, and can query statistics for emergency resources. The main functions include emergency information management, emergency material management, emergency material support, emergency equipment support, emergency resource query, etc. 


\section{6) Emergency Knowledge Management System}

Emergency knowledge was classified and managed according to the incident classification standard of maritime emergency. It maintained knowledge catalogues, such as laws, regulations, rescue knowledge and case files. Main functions: knowledge creation, keyword maintenance, knowledge inquiry, knowledge statistics and so on.

\section{7) Background Maintenance Management System}

The system management platform is mainly oriented to administrators, and manages the users, roles, privileges, logs, and configuration information. Management objects are managed in a hierarchical manner with administrators of different functions. Different administrators manage the system support software and application service through the system management platform. The main functions include: user management, organization management, role management, authority management, log management and so on.

\section{B. Pre-plan Intelligent Design}

Intelligent plan is the emergency plan automatically converted into the corresponding task set through a structured way to judge the alarm information, and automatic matched through the complete emergency resources according to the occurrence of unexpected events in different stages of evolution, so as to realize the automatic analysis of process execution of emergency plan. Real time reflected in the modern information technology integrated use of satellite positioning, geographic information technology, video surveillance, digital voice, to achieve a variety of emergency plan related resource information real-time dynamic tracking and scheduling, the formation of emergency rescue plan, combined with the actual situation of the original curing plan more flexible, vivid and efficient Express for emergency rescue command. As shown in figure 2.

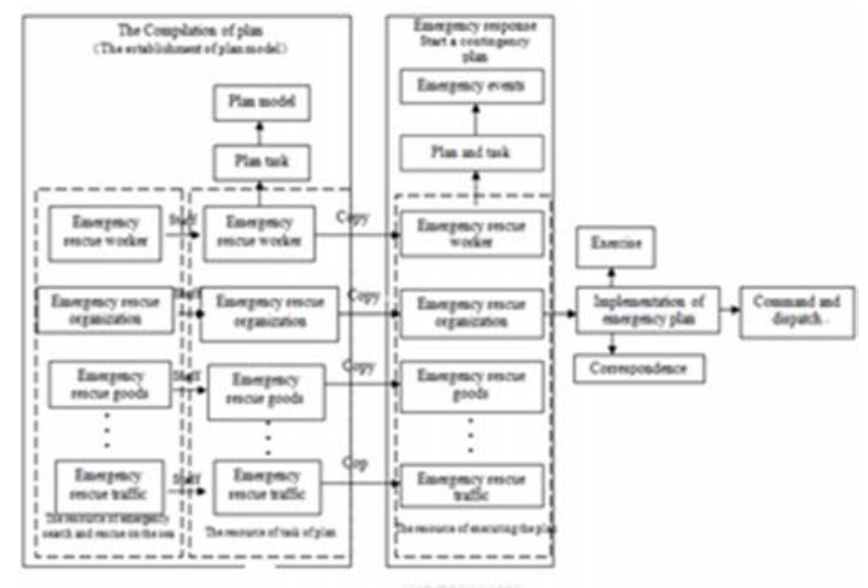

FIGURE II. INTELLIGENT DESIGN OF PREPLAN

\section{1) Set up a plan model}

The system provides a standard plan task and a resource data dictionary. Due to different accident grades and disaster types, different pre-plan models should be responded. When establishing a pre-plan model, relevant tasks for the pre-plan model from the standard plan task should be selected, and the corresponding emergency rescue resources for the task should be planned, so as to create a pre-plan model.

\section{2) Emergency Response}

In view of specific emergency events, a model of the corresponding response level is selected. By initiating the emergency plan, we automatically copy the model task and task resource information in the plan model to the emergency response structure, and form the emergency execution plan resources and plan tasks.

\section{3) Generating Execution Scheme}

According to the on-site feedback and experts' opinions, timely replenish, modify and improve the resources of emergency execution plan and tasks, and form a set of executable plans to deal with emergencies, so as to provide leadership decision-making and command scheduling.

\section{4) Command and Dispatch}

The core of the execution plan is task management and coordinated command. The plan model defines the information exchange interface, which enables real-time scheduling based on geographic information system, voice communication system and video surveillance system. In the process of command and dispatch, the tasks and resources can be temporarily adjusted, and the scheduling of the resources is completed until the emergency disposal of the whole event is completed.

\section{System Architecture Design}

The emergency command system can be divided into six layers according to the level: the basic environment layer, the system software layer, the data layer, the component and the interface layer, the application layer and the portal layer. As shown in figure 3 . The basic environment mainly for maritime emergency command system of intelligent hardware, including computers, storage devices, network environment, communications equipment (wired and wireless (3G)) and SMS platform; the system software layer includes relevant software and maritime emergency decision to use, including the operating system, database, middleware, application software GIS platform, software etc.. Data layer realizes data storage and processing, including event database, preplan database, statute database, case database (historical data + external case) and so on. Component and interface layer realizes compatibility between emergency command system and related software and hardware. The general assembly includes user management, SMS, telephone communication, GIS service access, and the interface module includes VTS exchange interface, ship dynamic system interface, GIS (GPS) AIS system interface, information transmission interface, meteorological information, hydrology, tide information interface; application layer security by programming the maritime intelligent emergency command system the realization of the function, including event response, disposal and disposal decision tracking verification, tracking and other functions. 


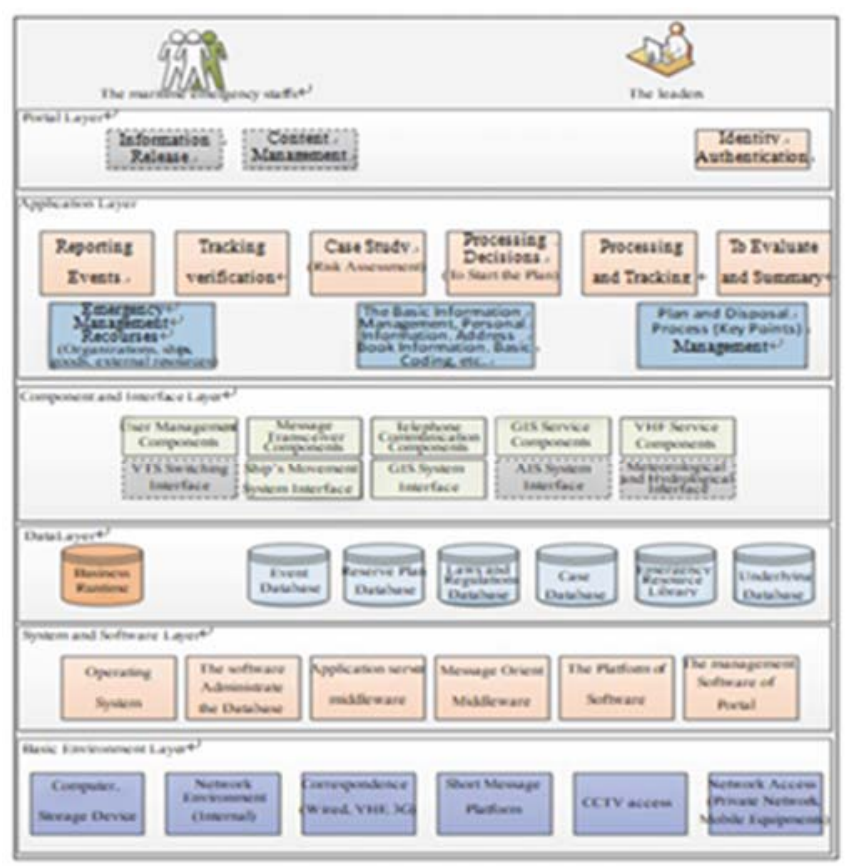

FIGURE III. THE SYSTEMATIC SYSTEM FRAMWORK

\section{System Technical Characteristics}

Maritime emergency command system on intelligent information integration and terminal integration, system design in accordance with the component model, considering the future expansion of the system; to provide a variety of interactive interface, meet the diversity of information, and based on GIS vector map, electronic chart, image in visual design. In terms of emergency rescue by way of example of the model, using the plan extraction model, the formation of the executive scheme through the dynamic model instantiation, execute structured task data in command scheduling, the implementation of the plan in the allocation of resources, to achieve the intelligent treatment plan. The system adopts the $\mathrm{B} / \mathrm{S}$ model, through load balancing, the support of a large number of visit Web. Design and development using JAVA language, using Struts+Hibernate pattern of development, enhance the reusability, scalability and security; ensure the stable operation of the system.

\section{CONCLUSION}

The present situation of the construction of the maritime emergency command was analyzed, and intelligent maritime emergency command system was established from four aspects of functional structure, intelligent pre-plan, system architecture, technical features, reflecting the functional completeness, technical feasibility, simple operation and high integration features, which is conducive to the maritime emergency command and intelligence information. So as to provide technical support to meet the needs of the rapid development of social economy for the water emergency management work.

\section{ACKNOWLEDGMENT}

This paper was supported by Zhejiang University Student Science and Technology Innovation Program and XinMiao Talents Program (2017R411057).

\section{REFERENCES}

[1] Devinder Grewal, Vinh V. Thai, The Maritime Security Management System[C]//Wang Zuwen, Proceedings of Globalization and MET, Dalian Maritime University Press, 2006, 37 - 54.

[2] Sen Ye, AIS and Long Term Management of Water Traffic Safety[C]// Qinglie $\mathrm{Li}$, Proceedings of The First Maritime Forum of Guangdong, Dalian Maritime University Press, 2006, 56-58.

[3] Z.L.Yang, S.Bonsall, Q.E.Fang, et al, Maritime Security-Assessment and Management[C]//Wang Zuwen, Proceedings of Globalization and MET, Dalian Maritime University Press, 2006, 326-342.

[4] Chuantang Yang, Jianzhong He, Dexing Song, et al, The 2013 Report on China Shipping Development[R].China Communication Press CO.,Ltd. Ministry of Transport of the People's Republic of China.2014.

[5] BiliangZhong, The Secondary Development and Application of VTS System[C]// Qinglie Li, Proceedings of The First Maritime Forum of Guangdong, Dalian Maritime University Press, 2006, 375-379. 